\title{
Influence of radiotherapy on blood pressure in patients with cervical and upper thoracic esophageal cancer
}

\section{Tai-wei Sun}

Zhongshan Hospital Fudan University

Hong-jie He

Lin Fen Central Hospital

\section{Yan-ming Zhang}

Lin Fen Central Hospital

\section{Shu-min Zhang}

Zhongshan Hospital Fudan University

\section{Xiao-chun Zhang}

Zhongshan Hospital Fudan University

\section{Li-jie Tan}

Zhongshan Hospital Fudan University

Zhao-Chong Zeng ( $\nabla$ zeng.zhaochong@zs-hospital.sh.cn )

Zhongshan Hospital Fudan University https://orcid.org/0000-0001-5085-6761

\section{Research}

Keywords: thyroid function; systolic blood pressure; diastolic blood pressure; radiotherapy

Posted Date: April 20th, 2020

DOl: https://doi.org/10.21203/rs.3.rs-22116/v1

License: (c) (i) This work is licensed under a Creative Commons Attribution 4.0 International License. Read Full License 


\section{Abstract}

Background An interesting clinical phenomenon occures frequently in our work, which indicates that blood pressure could return to normal in some hypertension patients with cervical \& upper thoracic esophageal cancer undergoing radiotherapy. We speculated that this may be related to thyroid gland damage by radiotherapy.

Methods We retrospectively analyzed pre- and post-radiotherapy (RT, 1.5months, 4.5 months and 7.5months) systolic blood pressure (SBP) and diastolic blood pressure (DBP) for a cohort of esophageal cancer patients. At 7.5 months post-RT, serum free triiodothyronine (FT3), free tetraiodothyronine (FT4) and thyroid-stimulating hormone (TSH) concentrations were also measured to evaluate the changes of thyroid hormones (THs) and its correaltion with changes of blood pressure. To confirm the influence of radiation on thyroid, patients were divided into cervical \& upper thoracic group (C\&U, n=55) and middle \& lower thoracic group (M\&L, $n=57)$.

Results In the C\&U group, the decreases $( \pm S D)$ in SBP and DBP, respectively, were $5.51 \mathrm{mmHg}( \pm 1.83$, $P=0.003)$ and $3.62 \mathrm{mmHg}( \pm 1.38, P=0.002)$ at 1.5 months post-RT, $6.73 \mathrm{mmHg}( \pm 2.24, P=0.001)$ and 6.15 $\mathrm{mmHg}( \pm 1.57, P=0.012)$ at $4.5 \mathrm{months}$ post-RT, and $8.02 \mathrm{mmHg}( \pm 2.73, P<0.001)$ and $7.91 \mathrm{mmHg}( \pm 2.33$, $P<0.001)$ at 7.5 months post-RT. The corresponding decreases in the M\&L group were not significant at any time post-RT. At 7.5 months post-RT, serum FT3 and FT4 concentrations in C\&U group decreased significantly $(P<0.05)$ from before RT to 7.5 months after the end of RT, and TSH concentration increased significantly after RT $(P<0.05)$. In addition, Pearson analysis showed that both FT3 and FT4 was positively correlated with decrease of systolic BP ( $r=0.430, P=0.001 ; r=0.667, P<0.001$, respectively $)$ and diastolic $\mathrm{BP}(\mathrm{r}=0.370, P=0.005 ; \mathrm{r}=0.469, P<0.001$ respectively $)$.

Conclusion The present study suggests that radiotherapy in patients with cervical and upper thoracic esophageal cancer might result in lowering BP, which might be related to thyroid gland damage by radiotherapy.

\section{Background}

Esophageal cancer is one of the most prevalent cancers in China. The incidence of esophageal cancer was $21.17 / 100$ thousand in China reported which ranked the fifth position, and the mortality rate was $15.58 / 100$ thousand, ranked at the fourth position among all malignant tumors in China [1]. Clinical therapeutic methods of esophageal cancer mainly include surgery, radiotherapy, and chemotherapy. A majority of patients undergoing radiotherapy were esophageal cancer in our institute. In the practice, we found that blood pressure could return to normal in some hypertension patients with cervical or upper thoracic esophageal cancer. We speculate that this phenomenon may be related to the radiation injury of thyroid gland.

During radiotherapy of esophageal cancer, especially for cervical or upper thoracic tumor segmentation, the proximity of the patient's thyroid gland to the radiation field leads to radiation injury for the patient's 
thyroid gland, which might cause hypothyroidism and affect the patient's quality of life [2]. Although thyroid dysfunction may influence blood pressure, the effect of radiotherapy for esophageal cancer on blood pressure has not been well established. In this study, all patients with esophageal cancer were treated with conformal intensity-modulated radiotherapy (IMRT) and were divided according to tumor segmentation into the cervical and upper thoracic (C\&U) group or the middle \& lower thoracic (M\&L) group. In fact, few radiation fields of patients with middle \& lower thoracic cancer extended to the height of thyroid gland, radiation injury of thyroid gland happens more easily in C\&U group than M\&L group. Thus, the influence of radiotherapy on blood pressure and thyroid function were evalueted through comparing the two groups. In addition, in order to confirm the role of radiation injury for thyroid gland in influencing blood pressure, we also evaluated the relationship between changes of thyroid hormones (THs) and changes of blood pressure before and 7.5 months after radiotherapy.

\section{Patients And Methods}

\section{Inclusion and exclusion criteria}

We included patients in this study based on the following inclusion criteria: 1) patients diagnosed with squamous cell carcinoma via pathological histology, 2) patients whose tumors were evaluated and given a TNM stage according to the eighth edition of the American Joint Committee on Cancer (AJCC)/Union for International Cancer Control (UICC) cancer staging manuals [3], 3) patients with a Karnofsky performance status (KPS) score $\geq 70$ points, and 4) patients undergoing RT due to low possibility of surgical resection.

We excluded patients from the study based on these exclusion criteria: 1) patients whose cases were complicated by other connective tissue diseases or metabolic endocrine diseases, 2) patients whose cases were complicated by severe hepatic or renal dysfunction, 3) patients with a family history of mental disease or psychosis, or 4) patients with distant metastasis definitively confirmed by imaging examination. This study was approved by the Ethics Committee of Zhongshan Hospital Affiliated to Fudan University, and the patients and their family members were informed and signed the informed consent.

In accordance with the eighth edition of the AJCC/UICC cancer staging manuals [3], we classified the esophageal cancer primary site according to typical endoscopic measurements of each region as measured from the incisors. Body size and height were also considered. Briefly, the cervical esophagus was defined as the region from esophagus inlet to sternal notch. The upper thoracic esophagus was defined as the region from the sternal notch to the lower border of the azygos vein, the middle thoracic esophagus as lower border of the azygos vein to lower border of the inferior pulmonary vein, and the lower thoracic esophagus as lower border of the inferior pulmonary vein to the stomach, including the esophagogastric junction. Location of cancer primary site was defined by cancer epicenter. Some patients with middle and lower thoracic esophageal cancer had supraclavicular nodes metastases, which may expose the thyroid to the target radiotherapy area. These patients were excluded from our study. 


\section{Treatment methods}

Patients were treated as follows: after simulating the radiotherapy treatment field using intravenous enhanced computed tomography (CT), patients were fixed in a supine position, and data obtained by CT scanning were imported to the 3D planning system (CMS XiO Treatment Planning System and Elekta Medical Systems). The gross tumor volume (GTV, the volume of lymph nodes $\geq 1 \mathrm{~cm}$ and visible tumor), clinical target volume (CTV, uniform external expansion of GTV for 5-7 mm, including superior mediastinal lymphatic drainage region and bilateral supraclavicular region) and planning target volume (PTV, including the scope and setup error of organ motion and CTV, namely external expansion of $\mathrm{x}$ - and $y$-axis for $10 \mathrm{~mm}$ and z-axis for $15 \mathrm{~mm}$ based on CTV with the geometric center of GTV as radiation field center) were delineated. The prescribed dose of PTV was 56-66 Gy (2 Gy/time, 5 times/week, a total of 28-33 times), and the treatment lasted for about 6 weeks. For all patients, platinum $\left(25 \mathrm{mg} / \mathrm{m}^{2}\right.$ of bodysurface area) and paclitaxel ( $50 \mathrm{mg} / \mathrm{m}^{2}$ of body-surface area) were administered intravenously for five cycles, starting on days 1, 8, 15, 22, and 29. All patients were treated in our department from March 2018 to July 2019.

Baseline BP were collected before the first radiotherapy appointment. Other BP data after RT were collected at three screening visits, at 1.5, 4.5, and 7.5 monts after the end of RT. All BP measurements were obtained by trained and certified observers who were masked to the intervention assignment. SBP was defined as the appearance of the first Korotkoff sound and DBP as the disappearance of the fifth Korotkoff sound. At each visit, three BP measurements were obtained while the patients rested quietly in a seated position for at least 5 min. Body weight and height were measured at a screening visit and BMI was calculated and used to exclude persons with severe obesity.

Serum free triiodothyronine (FT3), free tetraiodothyronine (FT4) and thyroid-stimulating hormone (TSH) concentrations were measured using standard blood draws; tests before RT were conducted before the first radiotherapy appointment, and those after RT were conducted at month 7.5 after the end of RT.

\section{Statistical analysis}

Statistical Product and Service Solutions (SPSS, IBM Corp.) version 16.0 was used for statistical analysis. Measurement data were expressed as mean \pm standard deviation. Student's $t$-test was used for the comparison of measurement data, paired $t$-test was used for the intragroup comparison before and after treatment, and Chi-square test $\left(\chi^{2}\right)$ was adopted for the comparison of enumeration data. Pearson correlation analysis was used to analyze the correlation between FT3, FT4, TSH and SBP, DBP. $P<0.05$ was considered to indicate a statistically significant difference.

\section{Results}

\section{Baseline characteristics}


The baseline characteristics of the 112 patients are presented in Table 1, according to segmentation of esophageal tumor. There were no statistically significant differences in clinical baseline data, such as sex, age, height, weight, smoking history, obesity, SBP, DBP, and hypertension between C\&U group and M\&L group ( $P>0.05)$ (Table1).

\section{C\&U and M\&L group changes in SBP, DBP after RT}

In the C\&U group, the decreases ( $\pm S D)$ in SBP and DBP, respectively, were $5.51 \mathrm{mmHg}( \pm 1.83, P=0.003)$ and $3.62 \mathrm{mmHg}( \pm 1.38, P=0.002)$ at 1.5 months post-RT, $6.73 \mathrm{mmHg}( \pm 2.24, P=0.001)$ and $6.15 \mathrm{mmHg}$ $( \pm 1.57, P=0.012)$ at 4.5 months post-RT, and $8.02 \mathrm{mmHg}( \pm 2.73, P<0.001)$ and $7.91 \mathrm{mmHg}( \pm 2.33$, $P<0.001)$ at 7.5 months post-RT. The corresponding decreases in M\&L group for SBP and DBP were, respectively, $2.03 \mathrm{mmHg}( \pm 1.32, P=0.223)$ and $2.25 \mathrm{mmHg}( \pm 1.07, P=0.218)$ at 1.5 months post-RT, 2.41 $\mathrm{mmHg}( \pm 0.97, P=0.081)$ and $2.53 \mathrm{mmHg}( \pm 0.82, P=0.236)$ at 4.5 months post $-\mathrm{RT}$, and $1.93 \mathrm{mmHg}( \pm 1.11$, $P=0.067)$ and $2.64 \mathrm{mmHg}( \pm 0.93, P=0.123)$ at 7.5 months post-RT. Details are shown in Table 2.

\section{C\&U and M\&L group changes in serum FT3, FT4 and TSH concentrations before and 7.5 months after RT}

In the C\&U group, serum FT3 levels dropped significantly from $4.19 \pm 1.15 \mathrm{pmol} / \mathrm{l}$ before RT to $1.92 \pm 1.45$ $\mathrm{pmol} / \mathrm{l}$ after RT $(\mathrm{t}=11.742, P<0.001)$. Similarly, serum FT4 concentrations decreased significantly from $14.79 \pm 3.23 \mathrm{pmol} / \mathrm{l}$ before RT to $8.11 \pm 4.01 \mathrm{pmol} / \mathrm{l}$ after RT ( $\mathrm{t}=19.041, P<0.001)$. Meanwhile, serum TSH concentrations increased significantly from $2.71 \pm 1.23 \mathrm{mlU} / \mathrm{I}$ before $\mathrm{RT}$ to $8.38 \pm 3.78 \mathrm{mlU} / \mathrm{I}$ after RT ( $\mathrm{t}=-11.195, P<0.001)$. In contrast for the M\&L group, the serum FT3, FT4 and TSH concentrations stayed relatively stable, at $4.09 \pm 1.31 \mathrm{pmol} / \mathrm{l}, 14.58 \pm 3.14 \mathrm{pmol} / \mathrm{I}$ and $2.76 \pm 1.14 \mathrm{mIU} / \mathrm{I}$ before $\mathrm{RT}$, and $4.13 \pm 1.32$ $\mathrm{pmol} / \mathrm{l}, 14.43 \pm 3.03 \mathrm{pmol} / \mathrm{l}$ and $2.71 \pm 1.09 \mathrm{mlU} / \mathrm{l}$ after RT, respectively. There were no significant differences in serum FT3, FT4 and TSH concentrations in the M\&L group comparing before RT and after RT concentrations $(P>0.05)$. Details are shown in Figure 1.

\section{Correlation between between changes of FT3, FT4 and changes of systolic, diastolic BP in C\&U group at}

\section{5 months post-RT}

Pearson analysis showed that both FT3 and FT4 was positively correlated with decrease of systolic BP ( $r=0.430, P=0.001 ; r=0.667, P<0.001$, respectively). Further analysis indicated a positive correlation between FT3ロFT4 and diastolic BP $(r=0.370, P=0.005 ; r=0.469, P<0.001$ respectively). Figures 2 show the dispersion between changes of FT3, FT4 and changes of systolic, diastolic BP.

However, no correlation was found between changes of TSH and changes of systolic, diastolic $\mathrm{BP}(\mathrm{r}$ $=-0.212, P=0.12 ; r=-0.279, P=0.059$, respectively). Figures 3 show the dispersion between changes of TSH and changes of systolic, diastolic BP.

\section{Discussion}

To our knowledge, few studies have explored the relationship between RT and blood pressure in patients with various locations of esophageal cancer. The present study was conducted to determine the effect of 
radiotherapy on $\mathrm{BP}$ and thyroid function in a cohort of patients with esophageal cancer. In this study, both SBP and DBP were significantly reduced in the study participants with cervical \& upper thoracic esophageal cancer. In contrast, BP was not significant reduced in those patients whose tumors lied in the middle \& lower thoracic segmentation. The possible reason for the difference between groups is that the thyroid gland is in the radiation field for $\mathrm{C} \& \mathrm{U}$ group patients, leading to radiation injury in the thyroid gland, causing hypothyroidism, which decreases blood pressure.

Radiotherapy can often lead to thyroid dysfunction, including hypothyroidism. Some studies have demonstrated that treatment of other cancers by RT can expose thyroid gland to high doses of radiation and increase TSH after radiotherapy [4-6]. Actually, in the treatment of radiotherapy for esophageal cancer, thyroid gland was usually not contoured on CT images taken for planning radiation therapy and the therapy was planned without treating the thyroid gland as an organ at risk [7]. Thus, all or part of the thyroid gland was included in the initial radiation field.

Nishiyama et al [8] demonstrated that in the early stage of radiotherapy, the permeability of cell membranes within the thyroid gland begins to increase, which leads to an increase in the level of thyroid hormone (FT3,FT4) released into the blood, and the pituitary gland is inhibited via negative feedback, thus reducing the level of TSH. After radiotherapy, changes in follicular cells in the thyroid gland, such as irreversible fibrosis and degeneration, gradually occur and the permeability of cell membrane also decreased so that FT3 and FT4 levels begin to drop and the level of TSH increases via negative feedback. For most patients in our study, athough concentration of FT3 and FT4 were still within normal limits at 7.5 montsh after post-RT, the decrease of FT3, FT4 and increase of TSH were be obviosly observed. This is in accordance with one previous study and known as subclinical hypothyroidism [7].

Thyroid hormones (THs) FT3 and FT4 have profound effects on cardiovascular functions, suggesting that THs may contribute to the development of elevated blood pressure. In fact, one previous study [9] have demonstrated that FT3 and FT4 are positively related to BP in euthyroid adults, but no significant relationship was found between TSH and elevated BP. This is in accordance with our findings. Multiple putative mechanisms could explain the relationship between THs and BP values. Firstly, a previous study showed that hyperthyroidism increases SBP by decreasing systemic vascular resistance, increasing heart contractility and heart rate, and raising cardiac output [10]. Secondly, FT4 may alter BP salt sensitivity [11] in euthyroid individuals, a factor considered physiologically relevant to the onset of hypertension. However, Itterman et al suggested that the effect of THs level variation on BP is only a direct, short-term effect, since only associations with current and not incidental hypertension were found in their study [12]. Thirdly, Konukoglu et al suggested that both subclinical and overt hyperthyroidism are related to endothelial dysfunction, which plays an important role in the pathogenesis of hypertension [13]. Furthermore, a previous study also showed that thyroid function, as measured by serum free FT4 and TSH levels, was intrinsically linked to variables of endothelial function, insulin sensitivity and vasodilation, in healthy euthyroid subjects [14]. Fourthly, a previous review suggested that hyperthyroidism and states of adrenergic hyperactivity have many common clinical features; several components of the cardiomyocyte $\beta$-adrenergic system are regulated by $\mathrm{TH}$, such as the $\beta 1$-adrenergic 
receptor, guanine nucleotide regulatory proteins, and adenylate cyclase [15]. The adrenergic system is the major regulator of cardiac and vascular function, and this is also accomplished through the activation of VEGF receptors located on the endothelial surface by local and systemic release of catecholamines [16].

In this study, patients were strictly screened according to inclusion and exclusion criteria, and there were no statistically significant differences between C\&U and M\&L groups in clinical baseline data, such as sex, age, height, weight, smoking history, thus ensuring the preciseness precision and reliability of the study. However, the present study has several imitations. Firstly, the organ-at-risk dose, especially for heart and thyroid, were not observed. Radiation-induced heart injury maybe an important factor affecting patient BP. In theory, the heart dose-volume measures in the M\&L group would be considerably higher than that in C\&U group, which can offset the influence of thyroid injury on BP. Thus, it is essential to distinguish the varying degrees of BP between heart function damage and thyroid injury. Secondly, this

study is retrospective and lacks long-term follow-up data, such as the changes of BP and thyroid hormones over 1 or 2 years. Thus, the research follow-up time should be extended to 1-2 years to observe the changes in BP and thyroid hormones for esophageal cancer patients who underwent RT.

\section{Conclusions}

In conclusion, the present study suggests that radiotherapy in patients with cervical and upper thoracic esophageal cancer might have a moderate effect in lowering BP. This phenomenon might be related to radiotherapy-induced damage of the thyroid gland. Clinical trials with a prospective design would provide a better opportunity to document any BP lowering effect. In addition, the treatment effect of radiotherapy in patients with hypertension and esophageal cancer should be evaluated.

\section{Abbreviations}

SBP, systolic blood pressure; DBP, diastolic blood pressure; RT, radiotherapy treatment; FT3, serum free triiodothyronine; FT4, free tetraiodothyronine; TSH, thyroid-stimulating hormone; C\&U, cervical \& upper thoracic; M\&L, middle \& lower thoracic; AJCC, American Joint Committee on Cancer; UICC, Union for International Cancer Control; IMRT, intensity-modulated radiotherapy; PET/CT, positron emission tomography/computed tomography; GTV, gross target volume; PTV, planning target; THs , Thyroid hormones; KPS, Karnofsky performance status

\section{Declarations}

\section{Ethics approval and consent to participate}

The study protocol was approved by the ethics board of Zhongshan Hospital, Fudan University, and all participants gave informed consent to participate.

\section{Consent for publication}


Not applicable.

\section{Availability of data and material}

The datasets used and/or analysed during the current study available from the corresponding author on reasonable request.

\section{Conflicts of interest}

The authors declare that they have no competing interests.

\section{Funding}

This work was supported by National Natural Science Foundation of China (No.U1505229) and the key program of Science and Technology Ministry (No. 2017YFC0112101).

\section{Authors' contributions}

Tai-wei Sun and Zhao-chong Zeng participated in research design, manuscript writing and revision. Taiwei Sun and Hong-jie He analyzed the data and revised the manuscript. Yan-ming Zhang, Shu-min Zhang, Xiao-chun Zhang and Li-jie Tan provided additional guidance and support for this research. All the authors have read and approved the final manuscript.

\section{Acknowledgments}

Not applicable.

\section{Author details}

${ }^{1}$ Department of Radiation Oncology, Zhong Shan Hospital, Fudan University, Shanghai, P.R. China

${ }^{2}$ Department of Oncology, Lin Fen Central Hospital, Shanxi, P.R. China

${ }^{3}$ Department of Cardiology, Zhongshan Hospital of Fudan University, Shanghai, P.R. China

${ }^{4}$ Department of Thoracic Surgery, Zhongshan Hospital of Fudan University, Shanghai, P.R. China

\section{References}

1. Chen W, Zheng R, Baade PD, Zhang S, Zeng H, Bray F, Jemal A, Yu XQ, He J. Cancer statistics in China, 2015. CA Cancer J Clin. 2016; 66:115-32.

2. Chen J, Su T, Lin Y, Wang B, Li J, Pan J, Chen C. .Intensity-modulated radiotherapy combined with paclitaxel and platinum treatment regimens in locally advanced esophageal squamous cell carcinoma. Clin Transl Oncol. 2018; 20: 411-419. 
3. Rice TW, Gress DM, Patil DT, Hofstetter WL, Kelsen DP, Blackstone EH. Cancer of the esophagus and esophagogastric junction-Major changes in the American Joint Committee on Cancer eighth edition cancer staging manual. CA Cancer J Clin.2017; 67(4):304-317.

4. Kuijpens JL, Nyklíctek I, Louwman MW, Weetman TA, Pop VJ, Coebergh JW. Hypothyroidism might be related to breast cancer in post-menopausal women. Thyroid. 2005;15(11):1253-9.

5. Dorri Giv M, Bahreini Toosi MH, Aghamiri SMR, Akbari F, Taeb S. Calculation of thyroid dose with planner system and evaluation of thyroid function after radiotherapy for patients with breast cancer. J Biomed Phys Eng. 2016;6(4):229-34.

6. Darvish L, Ghorbani M, Teshnizi SH, Roozbeh N, Seif F, Bayatiani MR, Knaup C, Amraee A. Evaluation of thyroid gland as an organ at risk after breast cancer radiotherapy: a systematic review and metaanalysis. Clin Transl Oncol.2018;20(11):1430-1438.

7. Ishibashi N, Maebayashi T, Aizawa T, Sakaguchi M, Okada M, Matsushita J. Computed Tomography Density Change in the Thyroid Gland Before and After Radiation Therapy. Anticancer Res.2018;38(1):417-421.

8. Nishiyama K, Kozuka T, Higashihara T, Miyauchi K, Okagawa K. Acute radiation thyroiditis. Int J Radiat Oncol Biol Phys .1996;36:1221-1224.

9. GuY, Zheng L, Zhang Q, Liu L, Meng G, Yao Z, Wu H, Xia Y, Bao X, Shi H, Wang H, Xu H, Sun S..Relationshipbetween thyroidfunction and elevated blood pressure in euthyroid adults. Journal of Clinical Hypertension. 2018; 20(10): 1541-1549.

10. Prisant LM, Gujral JS, Mulloy Hyperthyroidism: a secondary cause of isolated systolic hypertension. J Clin Hypertension. 2006;8(8):596-599.

11. Gumieniak O, Perlstein TS, Hopkins PN, Brown NJ, Murphey LJ, Jeunemaitre X, Hollenberg NK, Williams GH. Thyroid function and blood pressure homeostasis in euthyroid subjects. J Clin Endocrinol Metab.2004;89(7):3455-3461.

12. Ittermann T, Tiller D, Meisinger C, Agger C, Nauck M, Rettig R, Hofman A, Jørgensen T, Linneberg A, Witteman JC, Franco OH, Greiser KH, Werdan K, Döring A, Kluttig A, Stricker BH, Völzke H. High serum thyrotropin levels are associated with current but not with incident hypertension.

Thyroid.2013;23(8):955-963.

13. Konukoglu D, Uzun H. Endothelial dysfunction and hypertension. Adv Exp Med Biol. 2017 ;956:511-540.

14. Fernández-Real JM1, López-Bermejo A, Castro A, Casamitjana R, Ricart W. Thyroid function is intrinsically linked to insulin sensitivity and endothelium-dependent vasodilation in healthy euthyroid subjects. J Clin Endocrinol Metab.2006;91(9):3337-3343.

15. Klein I, Ojamaa Thyroid hormone and the cardiovascular system. N Engl J Med. 2001;344(7):501-509.

16. Izzo R, Cipolletta E, Ciccarelli M, Campanile A, Santulli G, Palumbo G, Vasta A, Formisano S, Trimarco $B$, laccarino $G$. Enhanced GRK2 expression and desensitization of beta AR vasodilatation in hypertensive patients. Clin Transl Sci.2008; 


\section{Tables}

Table 1.Baseline characteristics of the patients according to segmentation of esophageal cancer

\begin{tabular}{|c|c|c|c|}
\hline Factors & Cervical \& Upper Thoracic $(n=55)$ & Middle \& Lower Thoracic $(\mathrm{n}=57)$ & $P$ value \\
\hline re (years) & $54.37 \pm 7.62$ & $56.37 \pm 6.28$ & 0.125 \\
\hline \multicolumn{4}{|l|}{$\mathrm{x}$} \\
\hline Male & 49 & 46 & 0.294 \\
\hline Female & 6 & 11 & \\
\hline sight (cm) & $164.47 \pm 7.63$ & $165.22 \pm 6.75$ & 0.562 \\
\hline eight (kg) & $52.61 \pm 9.53$ & $54.27 \pm 8.56$ & 0.230 \\
\hline \multicolumn{4}{|c|}{ aoking history } \\
\hline Yes & 36 & 33 & 0.442 \\
\hline No & 19 & 24 & \\
\hline sesity (\%) & 34.2 & 40.1 & 0.212 \\
\hline P (mmHg) & $125.8 \pm 10.2$ & $123.6 \pm 6.9$ & 0.125 \\
\hline $3 \mathrm{P}(\mathrm{mmHg})$ & $82.3 \pm 5.8$ & $81.6 \pm 6.3$ & 0.091 \\
\hline $\begin{array}{l}\text { זpertension } \\
\text { (o) }\end{array}$ & 19.6 & 12.8 & 0.454 \\
\hline
\end{tabular}

sesity, body mass index $>30 \mathrm{~kg} / \mathrm{m}^{2}$; Hypertension, systolic blood pressure (SBP) $>140 \mathrm{mmHg}$ and/or diastolic ood pressure $(\mathrm{DBP})>90 \mathrm{mmHg}$ 
Table 2. Mean changes ( \pm SD) from baseline in SBP and DBP and body weight, according to segmentation of esophageal cancer

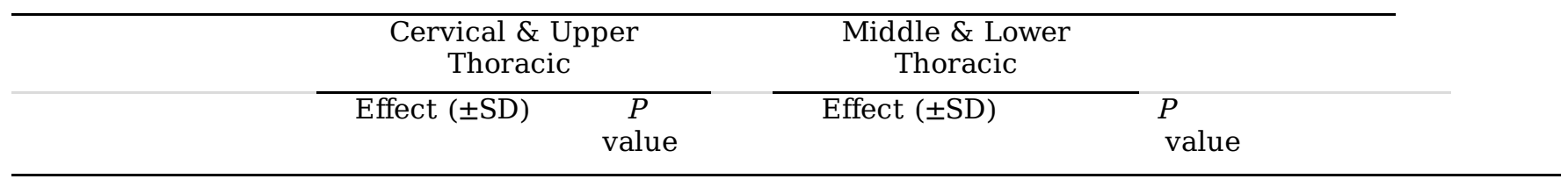

yes in SBP (mmHg)

\begin{tabular}{lcccr}
1.5 month & $-5.51( \pm 1.83)$ & 0.003 & $-2.03( \pm 1.32)$ & 0.223 \\
\hline 4.5 months & $-6.73( \pm 2.24)$ & 0.001 & $-2.41( \pm 0.97)$ & 0.081 \\
7.5 months & $-8.02( \pm 2.73)$ & $<0.001$ & $-1.93( \pm 1.11)$ & 0.067
\end{tabular}

yes in DBP (mmHg)

\begin{tabular}{lcccc}
1.5 month & $-3.62( \pm 1.38)$ & 0.002 & $-2.25( \pm 1.07)$ & 0.218 \\
\hline 4.5 months & $\begin{array}{c}-6.15 \\
( \pm 1.57)\end{array}$ & 0.012 & $-2.53( \pm 0.82)$ & 0.236 \\
& $-7.91( \pm 2.33)$ & $<0.001$ & $-2.64( \pm 0.93)$ & 0.123
\end{tabular}

yes in body weight

\begin{tabular}{lllcr}
\hline 1.5 month & $-1.33( \pm 1.12)$ & 0.294 & $-1.51( \pm 0.98)$ & 0.098 \\
\hline 4.5 months & $-1.15( \pm 0.84)$ & 0.161 & $-1.54( \pm 1.06)$ & 0.167 \\
\hline 7.5 months & $+1.31( \pm 0.77)$ & 0.312 & $+1.63( \pm 1.21)$ & 0.215
\end{tabular}

SBP, systolic blood pressure; DBP, diastolic blood pressure; SD, standard deviation.

\section{Figures}

A

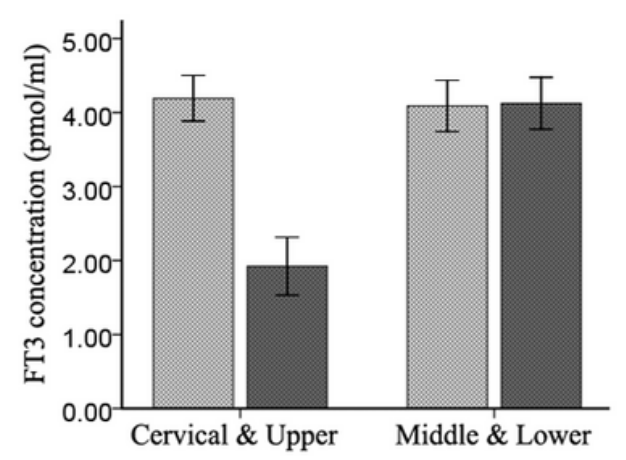

B

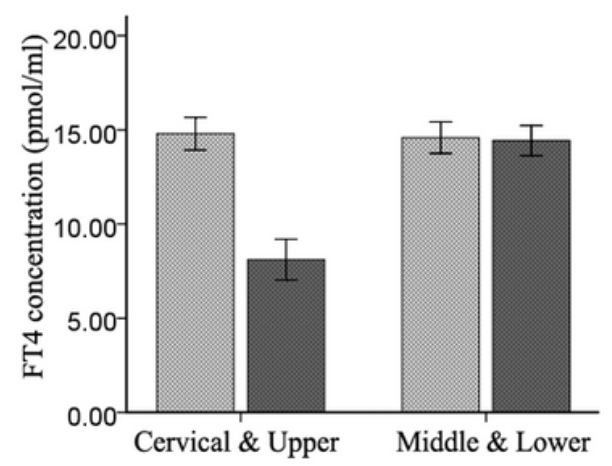

$\mathrm{C}$

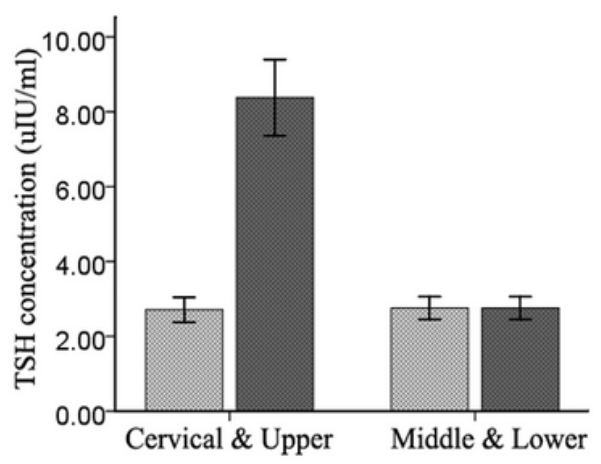




\section{Figure 1}

Comparison of serum free triiodothyronine (FT3), free tetraiodothyronine (FT4) and thyroid-stimulating hormone $(\mathrm{TSH})$ concentrations between cervical \& upper thoracic (C\&U) group and middle \& lower thoracic (M\&L) group before and 7.5 months after radiotherapy (RT). (A) Serum FT3 and (B) FT4 concentrations in C\&U group before RT were significantly higher than those after $R T$ ( $t=4.096, P<0.001$; $t=4.336, P<0.001)$, and $(C) T S H$ concentration was significantly lower after $R T(t=2.918, P=0.01)$. The M\&L group had no significant differences in serum (A) FT3, (B) FT4 and (C) TSH concentrations before RT compared to after RT ( $P>0.05)$.

A

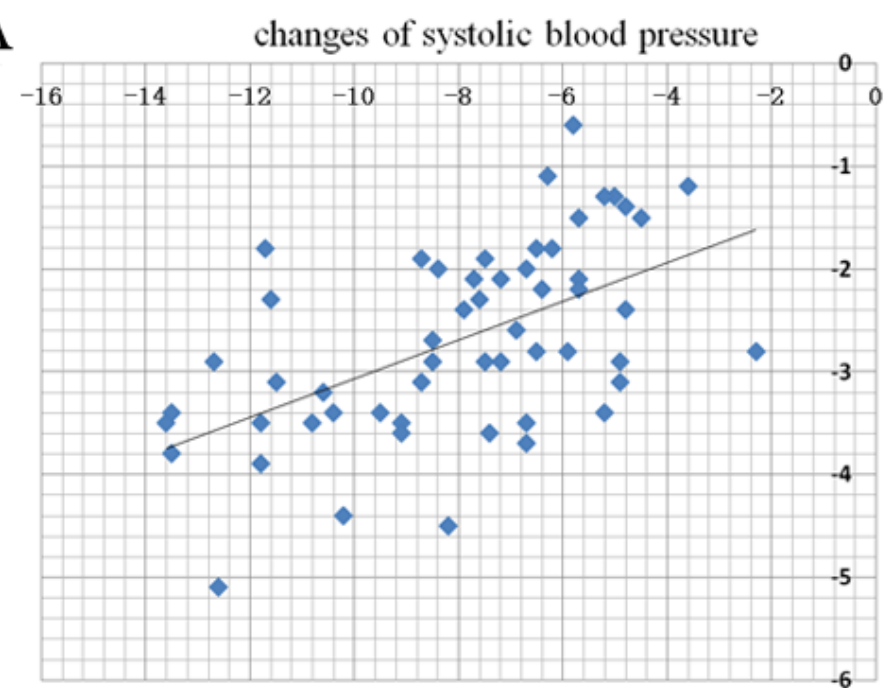

$\mathrm{C}$

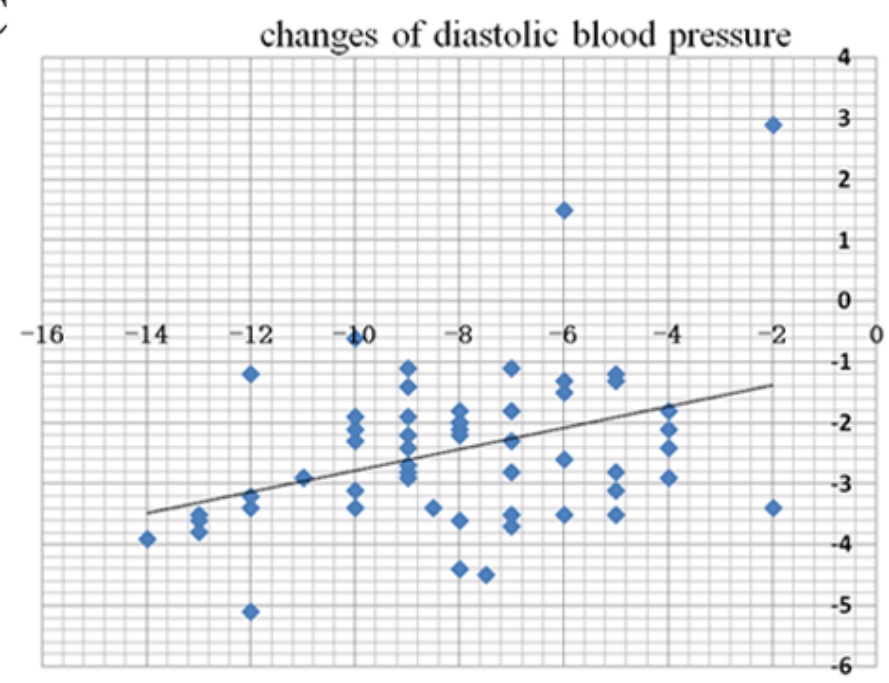

B changge of systolic blood pressure
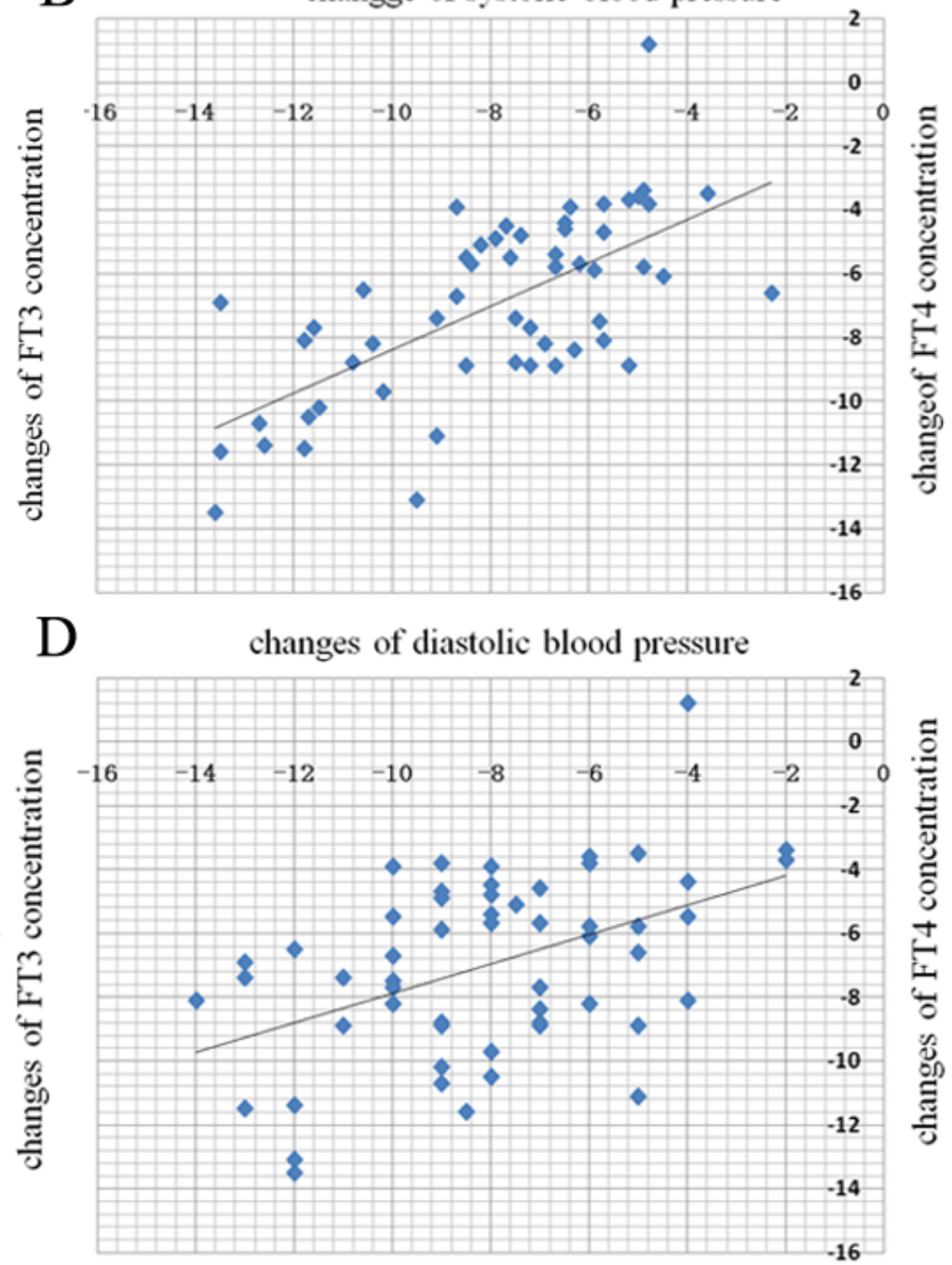

\section{Figure 2}

Correlation analysis of changes of FT3, FT4 and changes of systolic, diastolic BP in C\&U group. A: Pearson analysis demonstrated that changes of FT3 and systolic BP were positivelyly correlated $(r=$ $0.430, P=0.001) ; B$ : Pearson analysis revealed a positive correlation between changes of FT4 and systolic $\mathrm{BP}(r=0.667, \mathrm{P}<0.001)$; C: Pearson analysis indicated a positive correlation between changes of FT3 and 
diastolic BP $(r=0.370, P=0.005)$. $D$ : Pearson analysis indicated a positive correlation between changes of FT4 and diastolic BP $(r=0.469, P<0.001)$

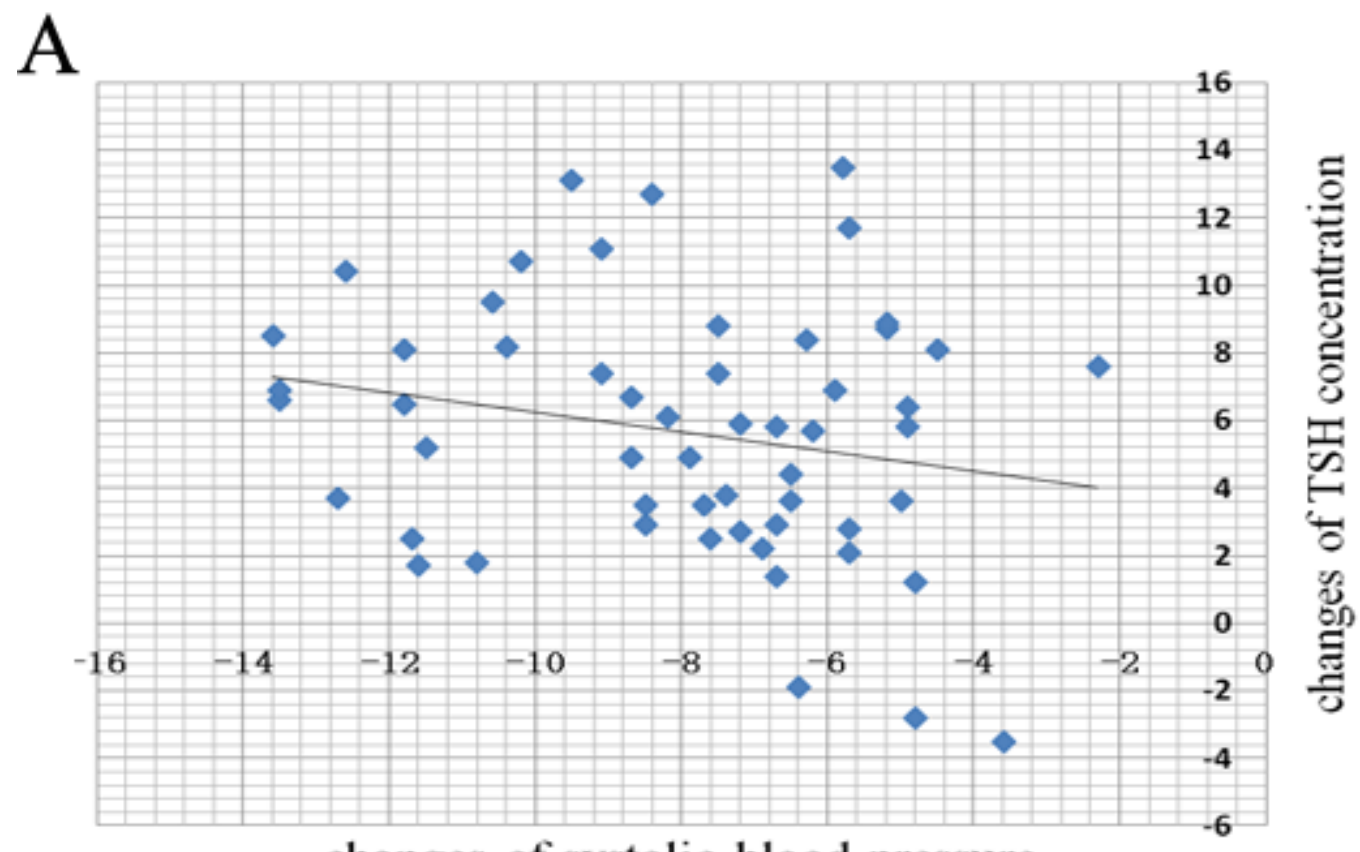

B

\section{changes of systolic blood pressure}

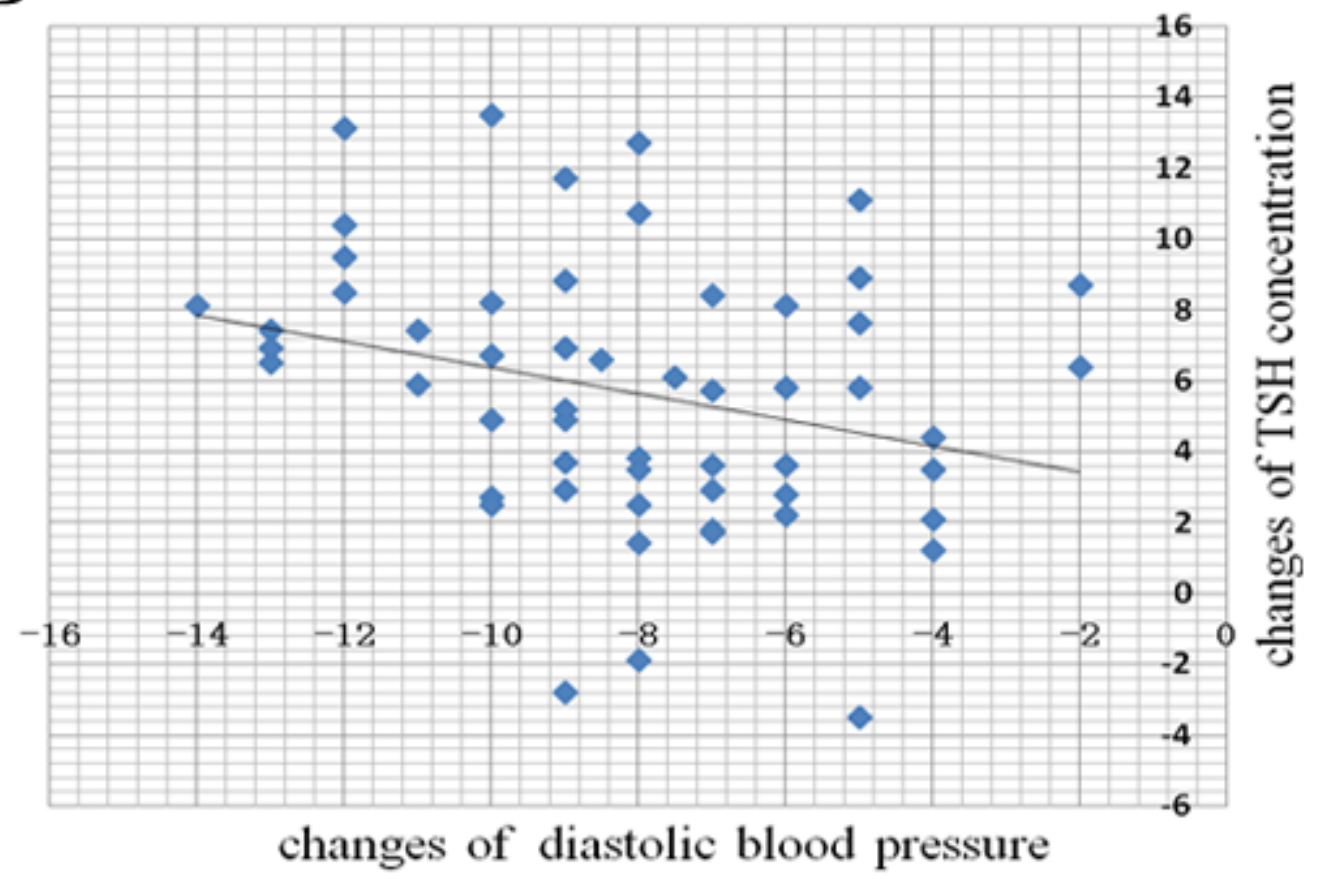

Figure 3

Correlation analysis of changes of TSH and changes of systolic, diastolic BP in C\&U group at 7.5 months after radioterapy. A: Neither positive nor negative correlation exists between changes of TSH and systolic BP $(r=-0.212, P=0.120)$; B: No correlation exists between changes of TSH and diastolic BP $(r=-0.279, P$ $=0.059)$. 